\title{
THE DEPENDENCE OF SOLAR MODULATION ON THE SIGN OF THE COSMIC RAY PARTICLE CHARGE
}

\author{
M. Garcia-Munoz, P. Meyer ${ }^{*}$, K.R. Pyle and J.A. Simpson* \\ Enrico Fermi Instıtute, University of Chicago, Chicago, I11inois \\ and \\ P. Evenson \\ Barto1 Research Foundation, University of Delaware, Newark, Delaware

\section{ABSTRACT}

We compare the solar modulation of galactic cosmic ray helium and electrons at $1 \mathrm{AU}$, within the 600-1000 MV magnetic rigidity interval, from 1965 through 1984. The time-intensity variations during the two solar maxima around 1970 and 1981 show that after 1970 the helium intensity recovers earlier than that of the electrons, whereas after 1981 the electron intensity recovers earlier than that of helium. The flux ratio of helium to electrons (He/e) undergoes a major increase during the 1969-1971 period and a major decrease during 1979-83. These experimental results can be interpreted as due to a dependence of the solar modulation of galactic cosmic rays on the sign of the particle charge, possibly as a consequence of drifts due to gradients and curvatures in the interplanetary magnetic field. However, the comparison of the shapes of the intensitytime curves of helium and electrons in the period 1970-1981 does not support a major specific prediction of the drift model.

1. Introduction. The perception that the interplanetary magnetic field is a distorted dipole, coherent in large scale, suggests that drifts due to field gradients and curvatures may play a significant role in solar modulation (Jokipii et al., 1977).

Because the drifts of particles of opposite charge move in opposite directions, the modulation effects on nuclei at a given point in the interplanetary space will be different from those on electrons.

This paper addresses the problem of the importance of drifts - trying to detect differences in the modulation of nuclear and electron cosmic rays that could be in principle attributed to the presence of drifts. We study the modulation of $70-95 \mathrm{MeV} / \mathrm{n}$ (739-866 MV) cosmic ray helium-4 and 600-1000 MeV cosmic ray electrons from 1965 through 1984 with special emphasis on the intervals around the two consecutive solar maxima in 1970 and 1981 in which the interplanetary magnetic field changed polarity.

2. The Experimental Data. Most of the experimental data used in this work have been obtained with the University of Chicago cosmic ray telescopes flown on balloons or aboard satellites.

Figure 1 shows the time dependence, within the 1960-1984 period of: (A) 27-day averages of the Climax neutron monitor counting rate, and; (B) 2-day averages of the 70-95 MeV/n helium-4 flux measured mostly by the U. of Chicago telescopes on IMP-3, 4, 5, 6 and 8 . The open circles 
are derived from the differential energy spectra and the near-continuous curve is derived from helium count rates identified by pulse height analys is.

Figure 2 shows the time dependence of the 600-1000 MeV cosmic ray electron flux. The open circles have been derived from differential energy spectra measured with experiments flown on balloons. The 1968-1971 solid curve represents

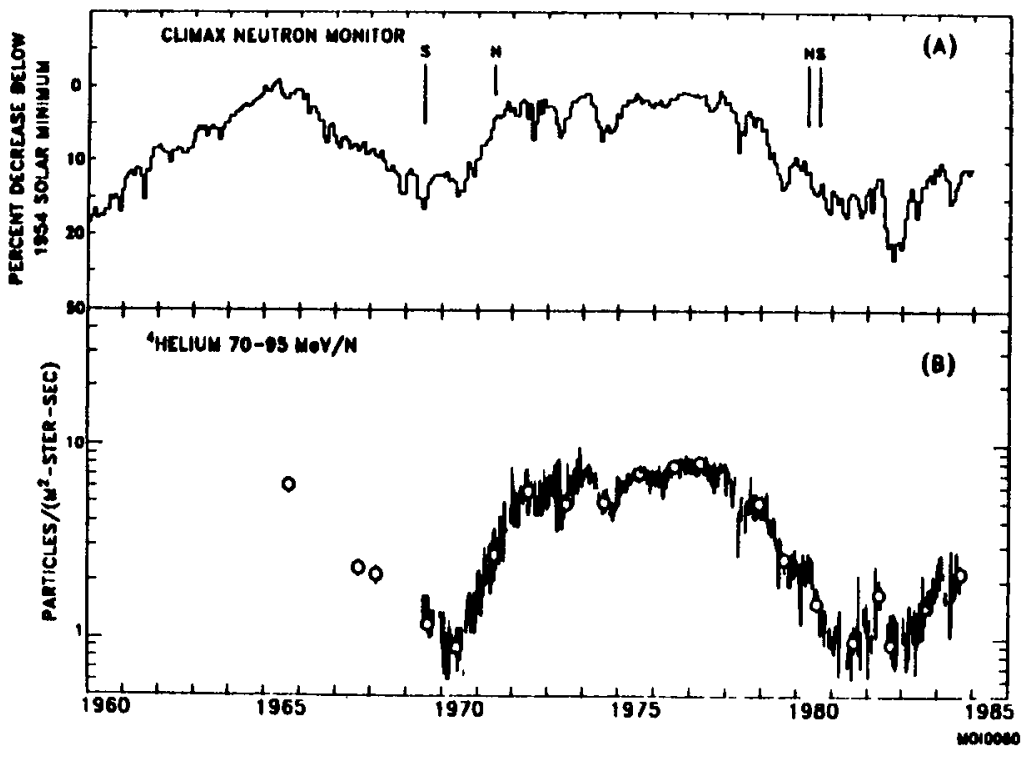

Figure 1

continuous measurements of 700-1000 MeV electrons measured on the satellite 0G0-5 (Burger and Swanenburg, 1973), normalized to the 1968-71 balloon measurements. The curve of small points from 1978 through 1984 are daily rates of $\sim 600-1000 \mathrm{MeV}$ electrons measured by the Chicago ISEE3 telescope (Evenson and Meyer, 1984). This curve can be normalized either to the 1982 or to the 1984 balloon measurements (the 1979 balloon point, measured during a Forbush decrease, cannot be used for normalization). Because the 1982 balloon flight data was taken not long after a sharp cosmic ray decrease we have normalized to the 1984 point. However, the conclusions reached in this paper are independent of which point, 1982 or 1984, is used for normalization. The arrows in Figures 1 and 2 point at the times when the solar polar magnetic fields reversed polarity (Howard, 1981).

3. The Relative Modulation of Nuclei and Electrons. An overview of the relative modulation of $70-95 \mathrm{MeV} / \mathrm{n}$ helium-4 and $600-1000 \mathrm{MeV}$ electrons is given in Figure 3, where the ratio He/e has been plotted as a function of time. Some of the ratios have been calculated using differentia 1 energy spectra of both helium and electrons (filled circles), while others have been calculated using different ia 1 energy spectra of helium and continuous satellite measurements of

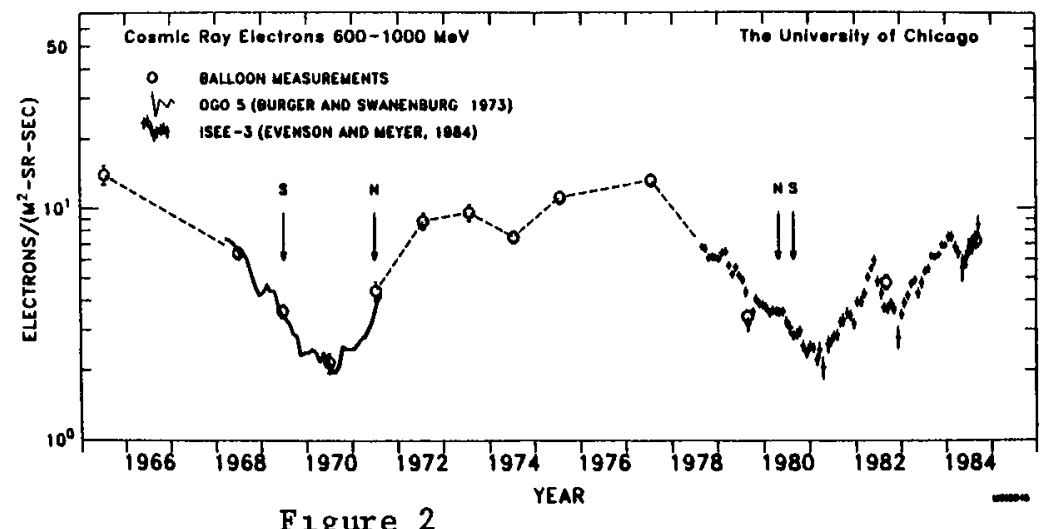


electrons (semifilled circles) and, final1y, others using continuous satellite measurements of both helium and electrons (open circles).

The dashed vertical lines mark the times at which the individual fluxes of 70-95 Mev/n helium and 600-1000 MeV electrons reached their minimum values during the two consecutive solar maxima. Again the arrows

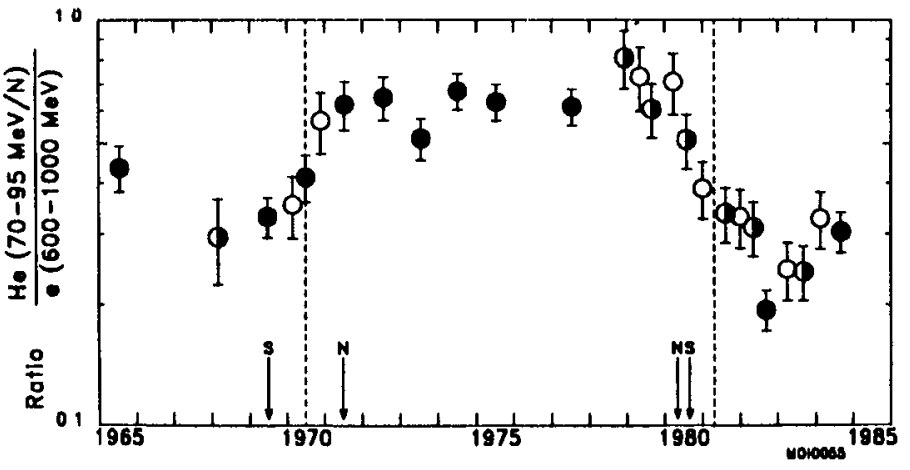

Figure 3 mark the times of polarity reversal of the solar polar magnetic fields.

Figure 3 clearly shows a difference in the modulation of cosmic ray particles of opposite charge during the two solar maxima. The ratio He/e increases from 1968-69 to 1971-72, while it decreases from 1979 to 198283. As can be seen in Figure 2, normalizing the ISEE-3 fluxes to the 1982 balloon point would further decrease the He/e ratio after 1977, except, of course, the ratio associated with the 1984 balloon point. Thus the opposite character of the time dependence of the ratios around the two solar maxima persists, regardless of the balloon point used to normalize the ISEE-3 data.

4. Discussion and Conclusions. As can be seen in Figures 1 and 2 , the periods 1971-73 and 1982-84 are similar time intervals in which the cosmic rays recover from the preceeding minimum intensity and increase towards the maximum associated with solar minimum activity. During these periods, in which the interplanetary magnetic field has opposite polarities, the ratio between the fluxes of cosmic ray helium and electrons, of nearly the same rigidity but opposite particle charge, differ by more than a factor 2. The fact that the electrons have higher velocity than the helium nuclei cannot alone explain the difference in the ratios, because any effect exclusively dependent on velocity is expected to be equally present in both compared periods.

The above facts suggest that a modulation process sensitive to the particle charge is operating in the interplanetary medium and an obvious candidate is drifts due to gradient and curvature in the interplanetary magnetic field as proposed by Jokipii et al. (1977).

However, although the results of the present work would be consistent with a significant role of drifts in solar modulation, they do not support one of the predictions of the recent drift model of solar modulation by Kota and Jokipii (1983). Their model predicts that, as a consequence of the predominant role played by drifts and the simultaneous presence of a wavy current sheet, the intensity of cosmic ray nuclei during the two halves of the 22-year magnetic cycle must have a very different time dependence, being relatively sharply peaked during the semicycle in which drifts bring positive particles from the equator to the heliospheric poles (e.g., around the 1965 solar minimum) and having a rather broad plateau during the semi-cycle in which drifts run in opposite direction (e.g., around the 1976 solar minimum). This is what is actually observed in the Climax neutron monitor counting rate for the solar minima of 1965 
and 1976 (Figure 1A). Since drifts run in opposite direction for negative particles, it is predicted that for cosmic ray electrons the shapes of the two semi-cycles should appear interchanged during the period 19591981. The data assembled in this work allow a check of this specific prediction for the 1970-1981 semicycle. Figure 4 shows a superposition of the time dependence of $70-95 \mathrm{MeV} / \mathrm{n}$ helium and $600-1000 \mathrm{MeV}$ electron fluxes both normalized to the years 1970 and 1977 . Note that from 1970 to 1980 the time dependence of the helium and electron fluxes have very closely the same shape, in disagreement with the model prediction.

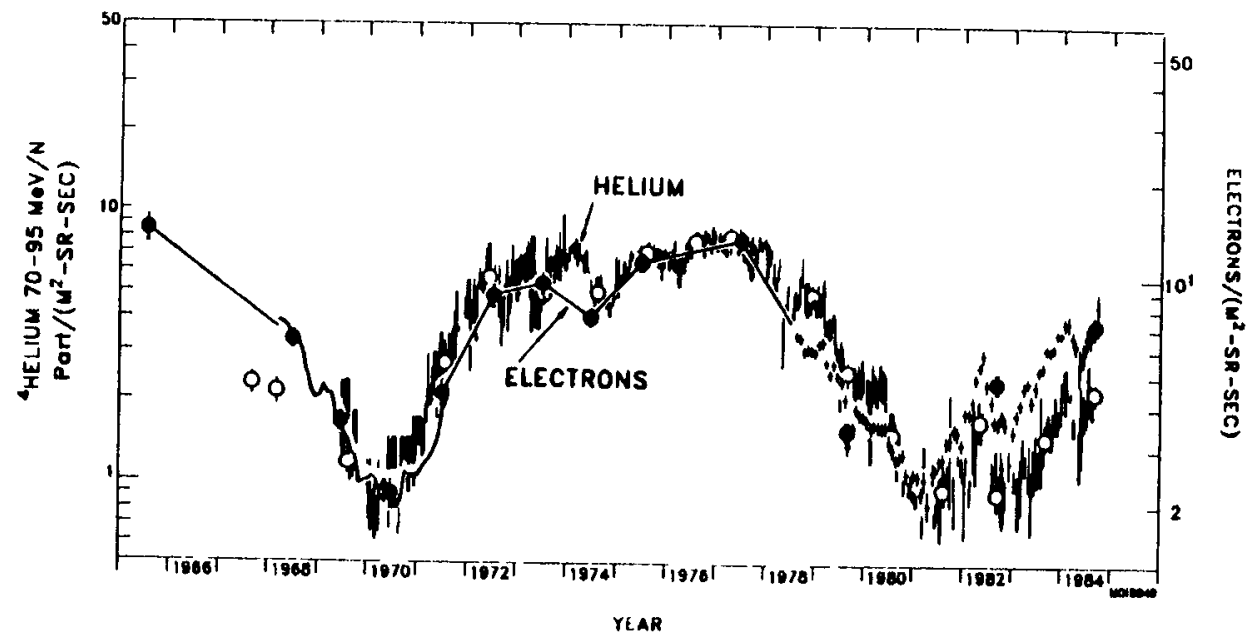

F1gure 4

5. Acknowledgements. We thank Scott Marusak and Leo Krawczyk for their assistance in the preparation of the data, and Leonard Shulman and Evelyn Tuska for their assistance in the 1984 balloon flight. This research was supported in part by NASA grants NGL 14-001-005, NGL 14-001-006 and NAG 5-374, NASA Contracts NAS 5-28442 and NAS 5-28500 and NSF Grants ATM 8412382, ATM 84-03718 and ATM 84-01427.

\section{References}

Burger, J.J. and Swanenburg, B.N., (1973), J. Geophys. Res., 78, 292. Evenson, P., and Meyer, P., (1984), Phys, Rev. Letters, 17, 329 . Howard, R., (1981), Smithsonian Ap. Obs. Spec . Rep. No. 393, 155. Jokipii, J.R., Levy, E.H. and Hubbard, W.B., (1977), AP.J., 213, 861. Kota, J., and Jokipii, J.R., (1983), Ap.J., 265, 573 . 